\title{
Exon skipping causes atypical phenotypes associated with a loss-of-function mutation in FLNA by restoring its protein function
}

\author{
Hirotsugu Oda ${ }^{1,2}$, Tatsuhiro Sato ${ }^{3}$, Shinji Kunishima ${ }^{4}$, Kenji Nakagawa ${ }^{1}$, Kazushi Izawa ${ }^{1}$, Eitaro Hiejima ${ }^{1}$, \\ Tomoki Kawai $^{\star, 1}$, Takahiro Yasumi ${ }^{1}$, Hiraku Doi ${ }^{1}$, Kenji Katamura ${ }^{1}$, Hironao Numabe ${ }^{1,5}$, Shinya Okamoto ${ }^{6,7}$, \\ Hiroshi Nakase $^{8}$, Atsushi Hijikata ${ }^{2}$, Osamu Ohara ${ }^{2,9}$, Hidenori Suzuki ${ }^{10}$, Hiroko Morisaki ${ }^{11}$, \\ Takayuki Morisaki ${ }^{11}$, Hiroyuki Nunoi ${ }^{12}$, Seisuke Hattori ${ }^{3}$, Ryuta Nishikomori ${ }^{1}$ and Toshio Heike ${ }^{1}$
}

Loss-of-function mutations in filamin A (FLNA) cause an X-linked dominant disorder with multiple organ involvement. Affected females present with periventricular nodular heterotopia (PVNH), cardiovascular complications, thrombocytopenia and EhlersDanlos syndrome. These mutations are typically lethal to males, and rare male survivors suffer from failure to thrive, PVNH, and severe cardiovascular and gastrointestinal complications. Here we report two surviving male siblings with a loss-of-function mutation in FLNA. They presented with multiple complications, including valvulopathy, intestinal malrotation and chronic intestinal pseudo-obstruction (CIPO). However, these siblings had atypical clinical courses, such as a lack of PVNH and a spontaneous improvement of CIPO. Trio-based whole-exome sequencing revealed a 4-bp deletion in exon 40 that was predicted to cause a lethal premature protein truncation. However, molecular investigations revealed that the mutation induced in-frame skipping of the mutated exon, which led to the translation of a mutant FLNA missing an internal region of 41 amino acids. Functional analyses of the mutant protein suggested that its binding affinity to integrin, as well as its capacity to induce focal adhesions, were comparable to those of the wild-type protein. These results suggested that exon skipping of FLNA partially restored its protein function, which could contribute to amelioration of the siblings' clinical courses. This study expands the diversity of the phenotypes associated with loss-of-function mutations in FLNA.

European Journal of Human Genetics (2016) 24, 408-414; doi:10.1038/ejhg.2015.119; published online 10 June 2015

\section{INTRODUCTION}

Filamin A (FLNA) is a large, dimeric, actin-binding protein that crosslinks actin filaments into orthogonal networks and links them to cell membranes. ${ }^{1}$ Each monomeric chain of FLNA has an N-terminal actin-binding domain and 24 immunoglobulin (Ig)-like repeats that are interrupted by two hinge domains. ${ }^{2}$ FLNA interacts with $>90$ binding partners and participates in various physiological processes, including cell migration, adhesion and signal transduction. ${ }^{1,3-5}$

FLNA is encoded by the FLNA gene (OMIM 300017, Xq28), which contains 48 exons and has a 7944-bp open reading frame (NM_001110556.1). In females, loss-of-function mutations in FLNA are associated with periventricular nodular heterotopia (PVNH), cardiovascular complications, thrombocytopenia and Ehlers-Danlos syndrome. ${ }^{6-10} \mathrm{PVNH}$ is characterized by the presence of nodules of neurons along the walls of the lateral ventricles, an inappropriate location caused by abnormal neuronal migration during cerebral cortex development. Cardiovascular complications are characterized by valvular insufficiency because of thickening of heart valves and a dilatation of large vessels. Thrombocytopenia associated with FLNA deficiency is characterized by giant platelets with anisocytosis and abnormal $\alpha$-granule distribution. Phenotypes of Ehlers-Danlos syndrome, including joint hypermobility, skin hyperextensibility and delayed scar formation, are observed among patients with loss-offunction mutations of FLNA. In males, however, loss-of-function mutations are typically embryonic lethal or cause death in early infancy because of severe heart failure and coagulopathy. To date, a number of rare male survivors have been reported. ${ }^{11}$ In addition to the symptoms observed in females, some of these male survivors demonstrate gastrointestinal complications, including intestinal malrotation and chronic intestinal pseudo-obstruction (CIPO). ${ }^{11-14}$

It is assumed that some male survivors carrying FLNA loss-offunction mutations retain sufficient FLNA protein expression to avoid the lethal effects; documented cases include splice-site mutations that produce both normal and aberrant FLNA mRNAs, ${ }^{15,16}$ and truncating mutations in the 5 ' end that result in translation initiating from a second, downstream start codon. ${ }^{12,14}$ Here, we present two male

${ }^{1}$ Department of Pediatrics, Graduate School of Medicine, Kyoto University, Kyoto, Japan; ${ }^{2}$ Laboratory for Integrative Genomics, RIKEN Center for Integrative Medical Sciences (RIKEN-IMS), Yokohama, Japan; ${ }^{3}$ Division of Biochemistry, School of Pharmaceutical Science, Kitasato University, Tokyo, Japan; ${ }^{4}$ Department of Advanced Diagnosis, Clinical Research Center, National Hospital Organization Nagoya Medical Center, Nagoya, Japan; ${ }^{5}$ Department of Genetic Counseling, Graduate School of Humanities and Sciences, Ochanomizu University, Tokyo, Japan; ${ }^{6}$ Department of Surgery (Hepato-Biliary-Pancreatic and Transplantation), Graduate School of Medicine, Kyoto University, Kyoto, Japan; ${ }^{7}$ Division of Pediatric Surgery, Kyoto University Hospital, Kyoto, Japan; ${ }^{8}$ Department of Gastroenterology and Hepatology, Graduate School of Medicine, Kyoto University, Kyoto, Japan; ${ }^{9}$ Department of Human Genome Research, Kazusa DNA Research Institute, Chiba, Japan; ${ }^{10}$ Department of Morphological and Biomolecular Research, Nippon Medical School, Tokyo, Japan; ${ }^{11}$ Department of Bioscience and Genetics, National Cerebral and Cardiovascular Center Research Institute, Osaka, Japan; ${ }^{12}$ Division of Pediatrics, Department of Reproductive and Developmental Medicine, University of Miyazaki, Miyazaki, Japan

*Correspondence: Dr T Kawai, Department of Pediatrics, Graduate School of Medicine, Kyoto University, 54, Kawahara-cho, Shogoin, Sakyo-ku, Kyoto, 606-8507, Japan. Tel: +81 75751 3291; Fax: +81 75752 2361; E-mail: tom0818@kuhp.kyoto-u.ac.jp

Received 2 March 2015; revised 22 April 2015; accepted 27 April 2015; published online 10 June 2015 
siblings with a 4-bp deletion in exon 40 . Within these siblings, inframe skipping of the mutated exon leads to the translation of functional FLNA protein, which could contribute to their survival. This report will broaden the understanding of the clinical impacts of exon skipping in genetic diseases and extend the known clinical spectrum of FLNA deficiency.

\section{MATERIALS AND METHODS}

\section{Whole-exome sequencing}

Genomic DNA from whole-blood samples taken from the patients and their parents were enriched for protein-coding sequences using TruSeq Exome Enrichment Kit (Illumina, San Diego, CA, USA), followed by massively parallel sequencing (HiSeq 1000, Illumina). Sequence data were mapped against the human reference genome (Genome Reference Consortium Human Build 37) using Burrows-Wheeler Aligner software. ${ }^{17}$ Variants were called using the Genome Analysis Toolkit. ${ }^{18}$ Annnotation of each variant was performed using an in-house program, then non-synonymous and splice-site variants with minor allele frequencies $>0.01$ were removed. All samples were collected with written informed parental consent, and the study protocol was approved by the ethical committee of Kyoto University Hospital, in accordance with the Declaration of Helsinki.

\section{Sanger sequencing}

Genomic DNA was amplified with LA Taq DNA polymerase (Takara, Shiga, Japan) and sequenced on an ABI 3700 (Applied Biosystems, Foster City, CA, USA). The primer sequences are listed in the Supplementary Table I. The identified variant of FLNA was deposited to http://databases.lovd.nl/shared/ genes/FLNA.

\section{RT-PCR}

To obtain T-cell lymphoblasts, the patients' peripheral blood mononuclear cells were stimulated with phytohemagglutinin (PHA, Sigma, St. Louis, MO, USA) and cultured in RPMI 1640 (Sigma) supplemented with $10 \%$ fetal calf serum and recombinant human IL-2 (Roche, Basel, Switzerland). Total RNA was extracted from T-cell lymphoblasts and reverse-transcribed using M-MLV Reverse Transcriptase (Invitrogen, San Diego, CA, USA). The resulting cDNA was PCR amplified with primer pairs (forward: 5'-CACAGAGCCAGGCAA CTACA-3', and reverse: 5'-CACATAAGCCACACCACAGG-3') specific for amplification of the cDNA sequence between exons 39 and 42 of FLNA. For the male siblings, PCR fragments were separated by agarose gel electrophoresis and Sanger sequenced.

\section{Western blot analysis}

Western blot analyses of extracts from the T-cell lymphoblasts and primary platelets were performed as described previously, ${ }^{19}$ using anti-FLNA monoclonal antibodies (MAB1678, Millipore, Darmstadt, Germany, and EP2405Y, Abcam, Cambridge, UK), anti- $\beta$-actin antibodies (ACTBD11B7, Santa Cruz Biotechnology, Dallas, TX, USA), and horseradish peroxidase-conjugated antimouse and anti-rabbit secondary antibodies (\#7076 and \#7074, Cell Signaling, Danvers, MA, USA). Images were obtained with an LAS-1000 mini (GE Healthcare, Pittsburgh, PA, USA) and processed using ImageJ. ${ }^{20}$

\section{RT-qPCR}

EBV-transformed lymphoblastoid cell lines (EBV-blasts) were established from the patients as previously described. ${ }^{21}$ RT-qPCR was performed using TaqMan Gene Expression Assays (Applied Biosystems) and a 7900HT Fast Real-Time PCR system (Applied Biosystems). Plasmids containing FLNA cDNAs were used as standards for absolute quantification of each cDNA, followed by normalization using $\beta$-actin as an internal control (4326315E, Applied Biosystems). Primer sequences were as follows: exon 40-non-skipped cDNA, forward: 5'-GCGTCGGGCTCCTTCAGT-3', reverse: 5'-CTGGTCACCTGGGC TGTCAT-3', and probe: 5'-AATCCCTGAAATTAGC-3'; exon 40-skipped cDNA, forward: 5'-CAACTACATCATCAACATCAAGTTTGC-3', reverse: 5'-TCACCTGGGCTGTCATATCCT-3', and probe: 5'-ACGTGCCTGAAATT-3'; and FLNA exon 37, forward: 5'-CTCGGGTCACAGGTGACGA-3', reverse:
5'-AGCAGGCTGAGATCCGTCTC-3', and probe: 5'-TGCGTATGTCCCACCT AAAGGTCGGCT-3'.

\section{Immunofluorescence analysis}

Peripheral blood smears were analyzed by immunofluorescence staining as described.22 In brief, peripheral blood smears were fixed in methanol, permeabilized with acetone and blocked with normal goat serum. The slides were concomitantly incubated with anti-FLNA (MAB1678, Millipore) and anti$\beta 1$-tubulin antibodies, ${ }^{23}$ and then reacted with Alexa Fluor 555-labeled antimouse IgG and Alexa Fluor 488-labeled anti-rabbit IgG (Invitrogen). The nuclei were counterstained with DAPI. Stained cells were examined using a BX50 fluorescence microscope (Olympus, Tokyo, Japan). Images were acquired using a DP70 digital camera and the DP Manager software (Olympus).

\section{Pull-down assay}

HEK293T cells expressing FLAG-tagged FLNA were lysed in $1 \mathrm{ml}$ of lysis buffer (50 mm Tris-HCl (pH 7.5), 1\% Triton X-100, $150 \mathrm{~mm} \mathrm{NaCl}$ and 1 mm EDTA) supplemented with $1 \times$ Protein Inhibitor cocktail. Ten micrograms of GST-ITG or GST bound to glutathione sepharose $4 \mathrm{~B}$ beads was incubated with the lysates for $2 \mathrm{~h}$ and washed three times with $1 \mathrm{ml}$ of the same buffer. Proteins were analyzed by SDS-PAGE, followed by western blotting using anti-FLNA (MAB1680, Millipore) and anti-GST (sc-138, Santa Cruz Biotechnology) antibodies.

\section{Reconstitution in M2 melanoma cells}

To create Myc-tagged FLNA constructs, wild-type and mutant ORF sequences of FLNA were subcloned into a pCMV-Tag3C vector (Stratagene, La Jolla, CA, USA). M2 cells expressing these constructs were selected with $800 \mathrm{mg} / \mathrm{ml}$ of G418 for 3 days on culture dishes and another 2 days on glass coverslips. The cells were fixed with $1 \%$ formaldehyde in PBS for $15 \mathrm{~min}$ and treated with $0.1 \%$ Triton X-100 in PBS for $15 \mathrm{~min}$. After blocking with $1 \%$ bovine serum albumin in PBS for $1 \mathrm{~h}$, the cells were incubated with anti-Myc (sc-40, Santa Cruz Biotechnology) and anti-vinculin (ab73412, Abcam) antibodies for $3 \mathrm{~h}$. Samples were washed three times with PBS for $5 \mathrm{~min}$ and incubated for $1 \mathrm{~h}$ with appropriate secondary antibodies. The samples were then mounted in Vectashield mounting medium (Vector Laboratories, Burlingame, CA, USA). Fluorescence images were obtained using an Olympus IX71 microscope equipped with a Hamamatsu ORCA-Flash4.0 CCD monochrome camera (Hamamatsu Photonics, Shizuoka, Japan) and subjected to image processing with MetaVue (Molecular Devices, Sunnyvale, CA, USA). The size of vinculinpositive focal adhesions was quantified using ImageJ software.

\section{Statistical analysis}

All the statistical analyses were performed using R version 3.0.1 (R Foundation for Statistical Computing, Vienna, Austria).

\section{RESULTS}

\section{Patient history}

Patient I is a 19-year-old male born at term with a birth weight of $3040 \mathrm{~g}$ to healthy, non-consanguineous Japanese parents. During the neonatal period, he suffered from intractable diarrhea and feeding difficulties, requiring parenteral nutrition. Radiographic examination revealed significant colonic distension and a delayed transit of contrast media, without any signs of intestinal obstruction, suggesting the diagnosis of CIPO. His abdominal symptoms gradually improved and parenteral nutrition was discontinued at 1 year of age. Abdominal pain with massive hematochezia occurred at 4 years of age. An exploratory laparotomy was performed, resulting in a diagnosis of intestinal malrotation, which was subsequently corrected using a Ladd's procedure. An intraoperative colonoscopy revealed multiple longitudinal ulcers in the terminal ileum, and a biopsy specimen revealed crypt disruption and mononuclear cell infiltration, suggesting the diagnosis of Crohn's disease. His abdominal symptoms improved with 
a

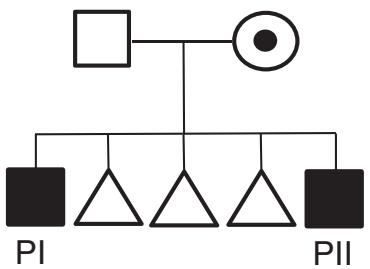

b

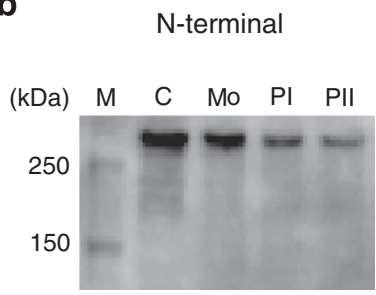

\section{C-terminal}

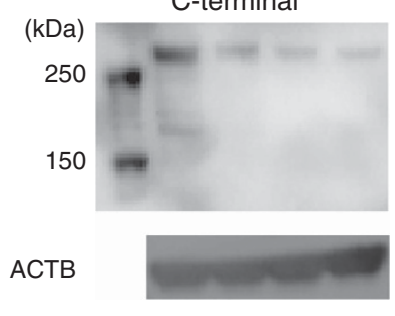

d

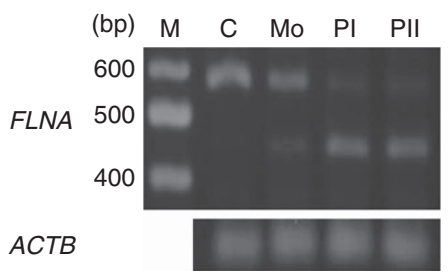

Normalized FLNA expression in

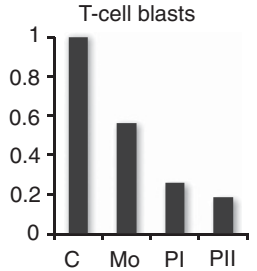

C Mo PI PII

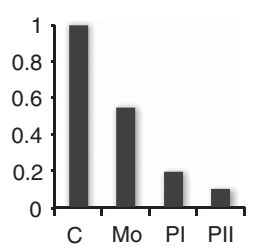

C

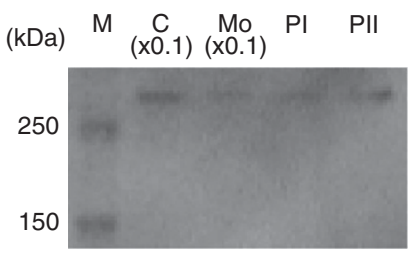

C-terminal

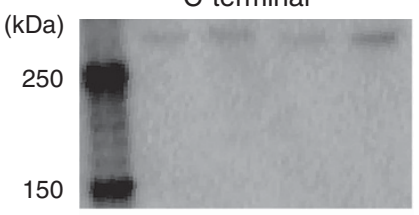

ACTB

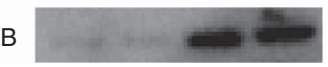

Normalized

FLNA expression in platelets
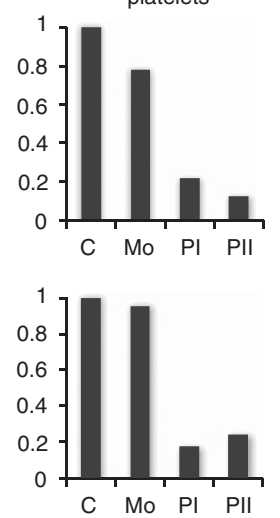

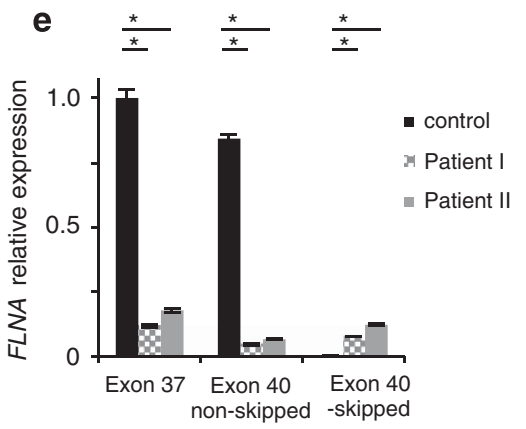

Figure 1 Molecular investigations of the pedigree (a) A pedigree of the family. PI and PII are the affected siblings. (b, c) Western blot analyses of the T-cell lymphoblasts (T-cell blasts; b) and platelets (c) from the siblings, their mother and a healthy control. $\mathrm{N}$ - and C-terminal regions of FLNA were stained separately, and the FLNA expression data were normalized with $\beta$-actin. In the platelet analysis (c), the samples of the control and the mother were both diluted 10-fold to improve FLNA expression analysis because of their exceedingly high expression as compared with the siblings' samples. Molecular weights (kDa) are shown on the left. ACTB, $\beta$-actin; C, healthy control; M, marker; Mo, mother. Shown is a representative of two experiments with consistent results. (d) RT-PCR analysis of FLNA mRNA. The mRNA of T-cell blasts from the siblings, their mother and the healthy control were reverse-transcribed, and a region between exons 39 and 42 was amplified by PCR. ACTB was used as a loading control. Shown is a representative of two experiments with consistent results. (e) Absolute quantification of FLNA mRNA, as well as mRNA with and without exon 40 skipping, isolated from EBV-blasts of the siblings. Primers specific for exon 37 were designed to quantify total FLNA expression, whereas two other sets were designed to quantify exon 40-skipped and non-skipped mRNA. Standard plasmids with known amounts of FLNA were used for absolute quantification of each mRNA. Results were normalized using $\beta$-actin as a control and are described as the ratio of the expression level to the level of exon 37 expression in the healthy control. Each experiment was performed in triplicate and the data are the mean \pm s.d. Shown is a representative of two experiments with consistent results. Statistical significance was determined by Student's $t$-test. ${ }^{*} P<0.0001$.

azathioprine and mesalamine treatment, but asymptomatic ileal ulcers remain to date.

Echocardiography revealed mitral valve prolapse with severe regurgitation, moderate aortic regurgitation and dilatation of the sinus of Valsalva. Computed tomography of the chest revealed moderate dilatation of both the ascending aorta and the bilateral pulmonary arteries. He had pectus excavatum and bilateral inguinal hernias. His skin was thin, but he did not have skin hyperextensibility or joint hyperlaxity. He exhibited occasional epistaxis and purpura on the lower extremities, along with mild thrombocytopenia. His psychomotor and cognitive development was normal, without signs of epilepsy, and a brain MRI in the neonatal period did not detect any structural abnormalities.

Patient II is 11 years old and the younger brother of patient I. He was born at 34 weeks of gestation, after a preterm labor, with a birth weight of $2154 \mathrm{~g}$. Soon after birth, he developed bilious vomiting and received a Ladd's procedure after the diagnosis of intestinal malrotation. However, after surgical correction, his bilious vomiting persisted 
Table 1 Clinical characteristics of the patients

\begin{tabular}{|c|c|c|c|c|c|}
\hline & Cardiac complications & Visceral abnormalities & $\begin{array}{l}\text { Neurological } \\
\text { abnormalities }\end{array}$ & $\begin{array}{l}\text { Skeletal } \\
\text { deformities }\end{array}$ & Other complications \\
\hline Patient I & $\begin{array}{l}\text { Severe MR with MVP moderate AR } \\
\text { dilatation of the sinus of Valsalva, } \\
\text { the ascending aorta and the } \\
\text { bilateral pulmonary arteries }\end{array}$ & $\begin{array}{l}\text { Intestinal malrotation } \\
\text { CIPO bilateral pneumothorax } \\
\text { Crohn's disease }\end{array}$ & None & $\begin{array}{l}\text { Pectus } \\
\text { excavatum }\end{array}$ & $\begin{array}{l}\text { Thrombocytopenia } \\
\text { bilateral inguinal hernias }\end{array}$ \\
\hline Patient II & $\begin{array}{l}\text { Moderate MR with MVP atrial } \\
\text { septal defect (spontaneously closed) }\end{array}$ & $\begin{array}{l}\text { Intestinal malrotation } \\
\text { CIPO }\end{array}$ & None & None & $\begin{array}{l}\text { Bilateral inguinal } \\
\text { hernias cryptorchidism }\end{array}$ \\
\hline
\end{tabular}

Abbreviations: AR, aortic regurgitation; CIPO, chronic intestinal pseudo-obstruction; MR, mitral regurgitation; MVP, mitral valve prolapse.

in the absence of any bowel-occluding region, suggesting the diagnosis of CIPO. After total parenteral nutrition for 9 months, episodes of feeding difficulties gradually disappeared. He was also affected with moderate mitral valve regurgitation and an atrial septal defect that closed spontaneously. He had bilateral inguinal hernias, but did not have other features of Ehlers-Danlos syndrome. His platelet count was normal, and he showed no bleeding tendency. He was neurologically intact and without epilepsy. A brain MRI at the age of 9 detected no abnormal findings. Pedigree information and clinical characteristics of the siblings are summarized in Figure 1a and Table 1.

The mother of these siblings is a 44-year-old woman. Except for three spontaneous abortions, she showed no apparent clinical presentations, including neurological and cardiovascular complications, and no bleeding tendency.

The cardiovascular complications of the siblings prompted us to suspect the diagnosis of Marfan syndrome, although genetic screening of FBN1, FBN2, TGFBR1, TGFBR2, ACTA2, FBLN4 and CHST14 could not detect any disease-causing mutations. Furthermore, arraybased comparative genomic hybridization could not detect any copy number variations.

\section{Genetic analysis}

We performed family-based whole-exome sequencing of the siblings and their parents. After filtering out common variants, we detected candidate variants in CLEC18A, COG8 and FLNA (shown in Supplementary Table II) in both siblings. Of these, a 4-bp deletion (NM_001110556.1:c.6425_6428delAGAG) in exon 40 of FLNA (NG_011506.1), a frameshift that is predicted to cause premature protein truncation (NP_001104026.1:p.(Glu2142AlafsTer22)), plausibly explained the siblings' clinical manifestations of valvulopathy and intestinal complications. We confirmed the variant by Sanger sequencing and determined that their mother is heterozygous for the variant (Supplementary Figure). No other variants in FLNA were identified by whole-exome sequencing or Sanger sequencing.

\section{Molecular investigations}

The 4-bp deletion in the FLNA gene was predicted to introduce a premature stop codon 65-bp downstream of the deletion site, producing a $234 \mathrm{kDa}$ truncated protein, which was not consistent with the non-lethal phenotype of the siblings. Therefore, we examined FLNA protein expression in PHA-induced T-cell lymphoblasts (T-cell blasts), as well as in the peripheral blood platelets of the siblings and their mother, using antibodies against the $\mathrm{N}$ - and $\mathrm{C}$-terminal regions of FLNA (FLNA-NT and -CT, respectively). The predicted $234 \mathrm{kDa}$ truncated protein was not detected by western blot in either of them. Instead, bands $>250 \mathrm{kDa}$ were detected by both FLNA-NT (MAB1678) and -CT (EP2405Y) antibodies (Figures $1 \mathrm{~b}$ and c). In both T-cell blasts and platelets, the FLNA accumulation of the mother was slightly less than that of a healthy control, whereas the patients had less than half of the control amount.

To elucidate the mechanism underlying the presence of a nearly full-length FLNA protein, we examined the subjects' FLNA cDNA by RT-PCR using primer pairs amplifying exons 39-42. Two fragments were obtained in the siblings (Figure 1d), and Sanger sequencing confirmed that the larger fragment was derived from the normal splicing of exon 40, which included the 4-bp deletion, whereas the smaller fragment resulted from an in-frame skipping of exon 40. These findings suggested that this variant induced both normal and alternative splicing of the mutated exon 40, and that the exon-skipped mRNA was translated through to the C-terminal region. Our preliminary reconstruction assay using a wild-type ORF and an ORF from an exon-skip mutant showed comparable expression of each length of protein, suggesting that the reduction of total FLNA protein in the siblings was not because of the instability of the mutant protein (data not shown). Next, we assayed the mutant mRNAs by quantitative RT-PCR using the siblings' EBV-transformed lymphoblastoid cell lines (EBV-blasts). We designed three primer sets: one specific for exon 37 (for the quantification of total FLNA), one for exon 40-nonskipped mutant cDNA and the other for exon 40-skipped cDNA. We conducted absolute quantifications using standard plasmids containing FLNA cDNAs. In both siblings, the amount of FLNA harboring exon 37 was markedly reduced, compared with that of a healthy control (Figure 1e). Furthermore, the siblings predominantly expressed exon 40-skipped FLNA in amounts greater than the healthy control. Taken together, these data indicate that the attenuated expression of mutant FLNA in the siblings was caused by the reduced quantity of mutant mRNA, and that exon skipping restored the nearly full-length FLNA protein, albeit lacking 41 internal amino acids (AAs).

To examine the function of the exon-skipped FLNA, we assayed the interaction between it and the GST-tagged cytoplasmic tail of integrin $\beta 7$ using a GST pull-down assay. The exon-skipped FLNA interacted more strongly with $\beta 7$ than wild-type FLNA (Figure 2a), which was consistent with a previous report. ${ }^{24}$ Furthermore, the truncated FLNA had a weaker interaction with $\beta 7$ than with the wild type. To further delineate the function of mutant FLNA in cells, we transfected Myctagged FLNA constructs into M2 melanoma cells naturally lacking FLNA expression and observed the development of focal adhesions. The focal adhesions induced by the truncated FLNA were smaller and disrupted, whereas those induced by the exon-skipped FLNA were comparable in size with those of the wild-type FLNA (Figure 2b). These data suggest that the skipping of exon 40 could restore the function of the translated product.

To further investigate the FLNA expression in each blood cell type, we performed immunofluorescence staining of lymphocytes, monocytes, granulocytes and platelets in the family. As shown in Figure 3a, the FLNA expression in each cell subset from the male siblings was 
reduced. In the mother's cells, the ratio of normal and reduced FLNAexpressing cells was not skewed (Figure 3b), suggesting that this variant did not lead to a growth advantage in blood cells.

\section{DISCUSSION}

Here, we report two surviving male siblings with a FLNA loss-offunction mutation that have cardiovascular and gastrointestinal complications. Although their atypical phenotype, including the spontaneous improvement of CIPO and the lack of PVNH, obscured the underlying genetic defect, whole-exome sequencing detected a 4-bp deletion in exon 40 . The exome sequencing also detected biallelic variants in two other genes (shown in Supplementary Table II), which have no reported associations to our cases' clinical phenotypes. Despite the prediction that this variant only produced a lethal truncated protein, a shorter protein, internally missing $41 \mathrm{AAs}$, was produced by in-frame skipping of the mutated exon 40.

Exon 40 of FLNA, which is skipped in the siblings, encodes 41 AAs, consisting of 5 AAs from the C-terminus of Ig19 (Gly2127-Ser2131) and 36 AAs from the N-terminus of Ig20 (Val2132-Pro2167). This alternative splicing of exon 40 was also found in cDNA libraries from human skeletal muscle and keratinocytes. ${ }^{25}$ Ig20-21 of FLNA has an important role in mechanotransduction. Several reports revealed that the $\mathrm{N}$-terminus of Ig20 forms a $\beta$-strand that interacts with, and occupies, the $\mathrm{C}$ and $\mathrm{D} \beta$-strands (known as the CD face) of the adjacent Ig21. ${ }^{26-28}$ When mechanical stresses stretch the FLNA protein, the masked CD face is exposed, increasing the affinity of Ig21 toward its binding partners, including integrins and migfilin, and leading to downstream signaling. ${ }^{29}$ In this study, the exon-skipped FLNA protein had stronger binding to $\beta 7$ and a similar capacity to induce focal adhesions, compared with that of wild-type FLNA. These results suggest that this alternative splicing partially restores FLNA protein function, which could result in the atypically mild phenotype of the siblings. Meanwhile, less expression and/or potential dysfunctions of the exonskipped FLNA protein may have caused their multi-organ involvement.

Most surviving males with FLNA loss-of-function mutations developed PVNH. Several reports explored the pathogenesis of PVNH; ${ }^{30,31}$ however, the precise pathogenesis of this disorder is still unclear. Our cases did not suffer from seizure episodes or developmental disabilities and did not have PVNH, according to a brain MRI. One explanation for the lack of these neurological abnormalities is that

a

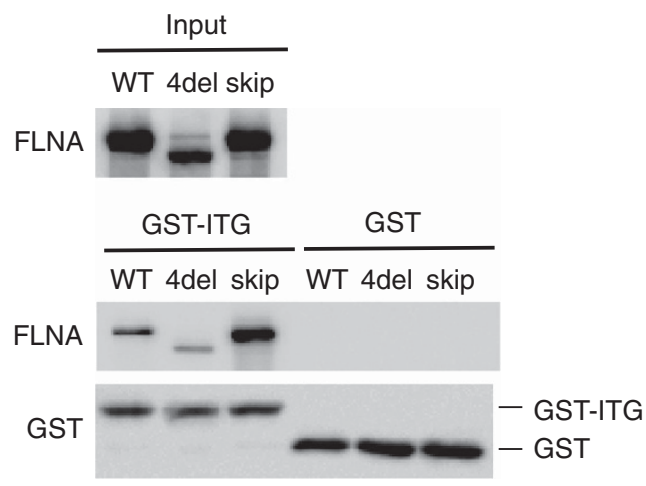

b
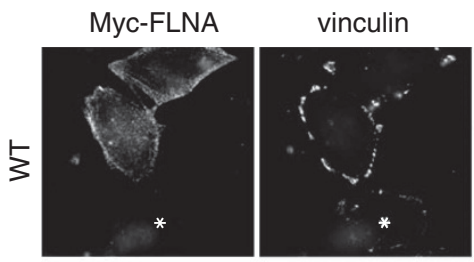

merge
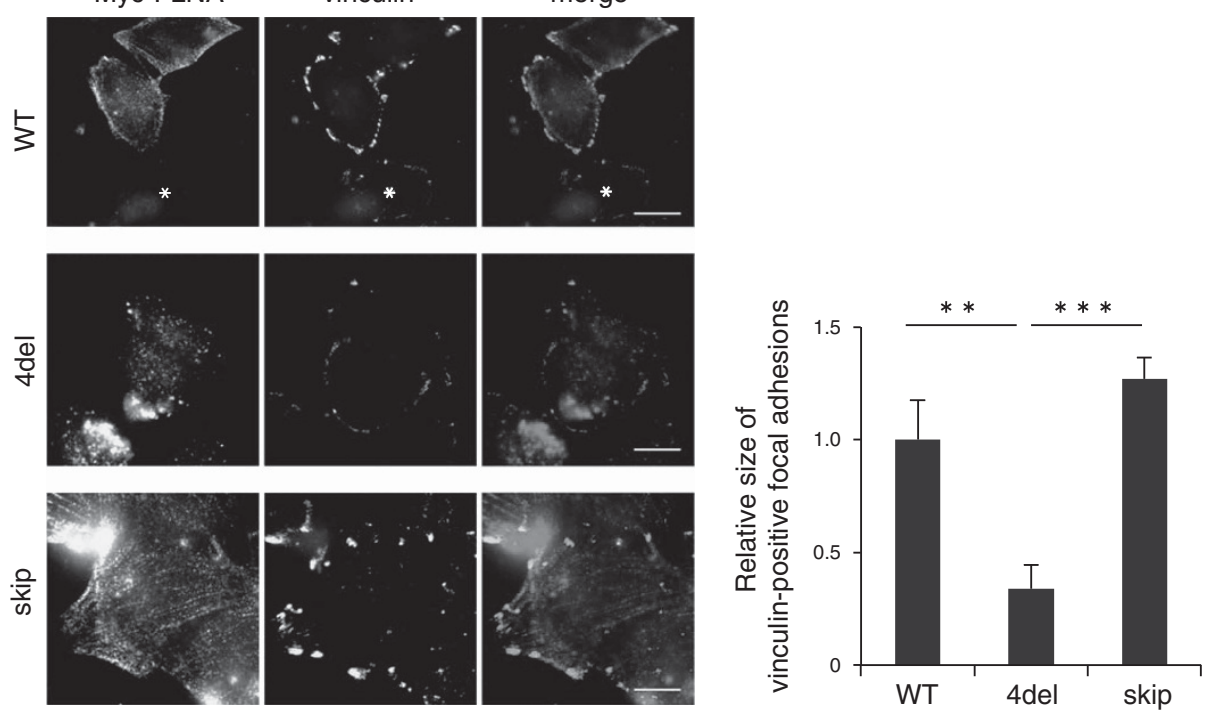

Figure 2 Functional analyses of the exon 40-skipped FLNA. (a) GST pull-down assay of FLNA with GST-fused Integrin $\beta 7$ (GST-ITG). WT, wild-type FLNA; 4del, truncated FLNA and skip, FLNA internally lacking 41 AAs. Representative results of three independent experiments are shown. (b) FLNA reconstitution assay in M2 cells. M2 cells expressing Myc-tagged wild-type, truncated or exon 40-skipped FLNA were stained with anti-Myc (red) and anti-Vinculin (green) antibodies. Myc-FLNA-negative M2 cells are indicated by an asterisk. Vinculin areas were quantified using ImageJ. Bars represent $20 \mu \mathrm{m}$. Each experiment was performed in triplicate, and the data are means \pm SEM. Statistical significance was determined by Student's $t$-test. ${ }^{* *} P<0.05,{ }^{* * *} P<0.01$. A full color version of this figure is available at the European Journal of Human Genetics journal online. 
a
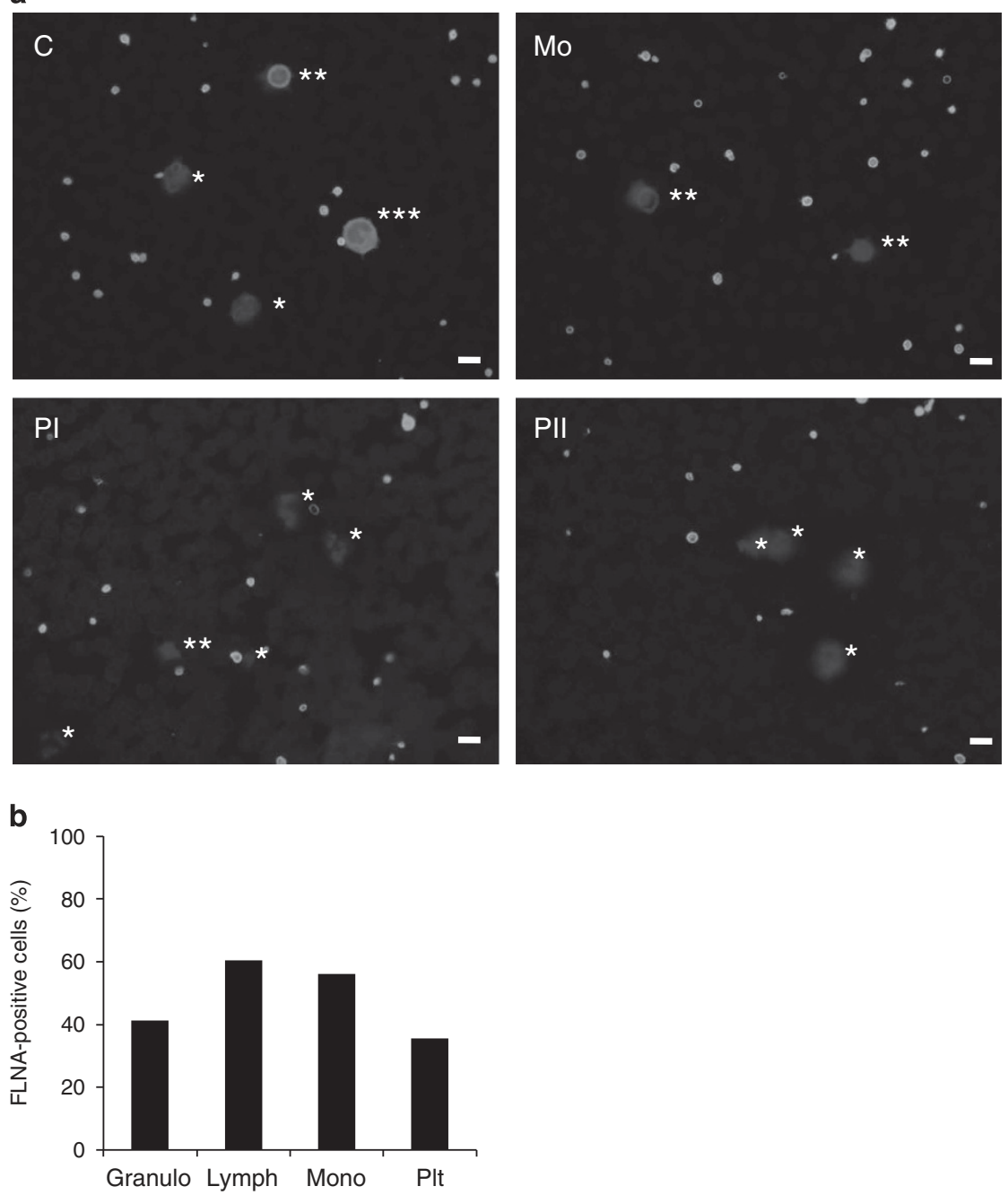

Figure $3 \mathrm{Immunofluorescence} \mathrm{staining} \mathrm{of} \mathrm{FLNA} \mathrm{in} \mathrm{blood} \mathrm{cells.} \mathrm{(a)} \mathrm{Peripheral} \mathrm{blood} \mathrm{smears} \mathrm{from} \mathrm{the} \mathrm{siblings} \mathrm{(PI} \mathrm{and} \mathrm{PII),} \mathrm{their} \mathrm{mother} \mathrm{(Mo)} \mathrm{and} \mathrm{a} \mathrm{healthy}$ control (C) were stained with anti-FLNA (red), anti- $\beta 1$-tubulin (green) and DAPI (blue). Merged images are shown. ${ }^{*}$ Granulocytes, ${ }^{* *}$ lymphocytes and ***monocytes. Image acquisition conditions were identical for all images. Data are representative of two independent experiments with consistent results. Note that there are two populations of platelets and lymphocytes in the mother: those with and those without FLNA expression. Bars represent $20 \mu \mathrm{m}$. (b) Percentage of FLNA-positive blood cells in the mother. Granulo, granulocytes; Lymph, lymphocytes; Mono, monocytes; PIt, platelets. A full color version of this figure is available at the European Journal of Human Genetics journal online.

the amount of exon-skipped FLNA may be greater in the siblings' brain tissues. Another possibility is that the exon-skipped FLNA may actively prevent the development of PVNH. As mentioned above, the $\mathrm{N}$-terminus of Ig20 was reported to mask Ig21, and indeed, the affinity of exon-skipped FLNA to integrin was higher than that of wildtype FLNA.

CIPO is a severe digestive syndrome characterized by intestinal dysmotility, resembling mechanical obstruction in the absence of any obstructive process. $^{32}$ Affected children generally present with recurrent episodes of abdominal pain, abdominal distension and inability to defecate, and require a long-term parenteral nutrition and/or a gastroenterostomy. The clinical course of CIPO is almost inevitably severe, owing to deteriorating bowel function and complications related to parenteral nutrition and surgery. ${ }^{32}$ CIPO was detected in a number of males harboring deleterious FLNA mutations. Furthermore, many male siblings of these patients die in early infancy because of gastrointestinal failure. ${ }^{11-14}$ Compared with these patients, the spontaneous remission of CIPO and the unreported complication of Crohn's disease in our cases are quite atypical. It is possible that other atypical FLNA-deficient patients have been overlooked or misdiagnosed, which suggests the need to re-define the phenotypic diversity associated with FLNA deficiency.

In conclusion, the in-frame skipping of the mutated exon causes atypical phenotypes associated with the loss-of-function mutation in FLNA, which broadens our understanding of the clinical impacts of exon skipping in genetic diseases and extends the known clinical spectrum of FLNA abnormalities. Further analysis is necessary to reveal the pathophysiological functions of the FLNA protein in each tissue.

\section{CONFLICT OF INTEREST}

The authors declare no conflict of interest. 


\section{ACKNOWLEDGEMENTS}

We thank the patients and their family members for participating in our study, Ms Yuki Takaoka for her technical assistance and Dr Hisanori Horiuchi for his kind advice on platelet analysis. This work was supported by grants from the Japanese Ministry of Education, Culture, Sports, Science, and Technology, and the Japanese Ministry of Health, Labor, and Welfare.

\section{AUTHOR CONTRIBUTIONS}

$\mathrm{HO}$ performed genetic and molecular analyses, and wrote the paper. TS, KN, $\mathrm{KI}, \mathrm{EH}, \mathrm{H}$ Nunoi and $\mathrm{SH}$ performed molecular investigations of the FLNA mutation. HS and SK performed imaging analysis of blood cells. H Numabe performed genetic counseling and performed genetic analyses. AH and $\mathrm{OO}$ performed whole-exome sequencing. HM and TM performed genetic analysis of Marfan syndrome-related genes and whole-exome sequencing. HD, KK, SO and $\mathrm{H}$ Nakase participated in the patients' diagnosis and contributed to writing the manuscript. TK, TY, RN and TH designed the study and contributed to writing the manuscript.

1 Zhou AX, Hartwig JH, Akyurek LM: Filamins in cell signaling, transcription and organ development. Trends Cell Biol 2010; 20: 113-123.

2 Nakamura F, Osborn TM, Hartemink CA, Hartwig JH, Stossel TP: Structural basis of filamin A functions. J Cell Biol 2007; 179: 1011-1025.

3 Ehrlicher AJ, Nakamura F, Hartwig JH, Weitz DA, Stossel TP: Mechanical strain in actin networks regulates FilGAP and integrin binding to filamin A. Nature 2011; 478: 260-263.

$4 \mathrm{Kim} \mathrm{H}$, McCulloch CA: Filamin A mediates interactions between cytoskeletal proteins that control cell adhesion. FEBS Lett 2011; 585: 18-22.

5 Nakamura F, Stossel TP, Hartwig JH: The filamins: organizers of cell structure and function. Cell Adh Migr 2011; 5: 160-169.

6 Fox JW, Lamperti ED, Eksioglu YZ et al: Mutations in filamin 1 prevent migration of cerebral cortical neurons in human periventricular heterotopia. Neuron 1998; 21: $1315-1325$.

7 Parrini E, Ramazzotti A, Dobyns WB et al: Periventricular heterotopia: phenotypic heterogeneity and correlation with filamin A mutations. Brain 2006; 129: 1892-1906.

8 Reinstein E, Frentz S, Morgan T et al: Vascular and connective tissue anomalies associated with X-linked periventricular heterotopia due to mutations in Filamin A. Eur J Hum Genet 2013; 21: 494-502.

9 Kyndt F, Gueffet JP, Probst V et al: Mutations in the gene encoding filamin A as a cause for familial cardiac valvular dystrophy. Circulation 2007; 115: 40-49.

10 Nurden P, Debili N, Coupry I et al: Thrombocytopenia resulting from mutations in filamin A can be expressed as an isolated syndrome. Blood 2011; 118: 5928-5937.

11 Oegema R, Hulst JM, Theuns-Valks SD et al: Novel no-stop FLNA mutation causes multi-organ involvement in males. Am J Med Genet A 2013; 161: 2376-2384.
12 Gargiulo A, Auricchio R, Barone MV et al: Filamin A is mutated in X-linked chronic idiopathic intestinal pseudo-obstruction with central nervous system involvement. Am J Hum Genet 2007; 80: 751-758.

13 Kapur RP, Robertson SP, Hannibal MC et al: Diffuse abnormal layering of small intestinal smooth muscle is present in patients with FLNA mutations and $x$-linked intestinal pseudo-obstruction. Am J Surg Pathol 2010; 34: 1528-1543.

14 van der Werf CS, Sribudiani Y, Verheij JB et al: Congenital short bowel syndrome as the presenting symptom in male patients with FLNA mutations. Genet Med 2013; 15: 310-313.

15 Guerrini R, Mei D, Sisodiya S et al: Germline and mosaic mutations of FLN1 in men with periventricular heterotopia. Neurology 2004; 63: 51-56.

16 Hehr U, Hehr A, Uyanik G, Phelan E, Winkler J, Reardon W: A filamin A splice mutation resulting in a syndrome of facial dysmorphism, periventricular nodular heterotopia, and severe constipation reminiscent of cerebro-fronto-facial syndrome. J Med Genet 2006; 43: 541-544.

$17 \mathrm{Li} \mathrm{H}$, Durbin R: Fast and accurate short read alignment with Burrows-Wheeler transform. Bioinformatics 2009; 25: 1754-1760.

18 McKenna A, Hanna M, Banks E et al: The Genome Analysis Toolkit: a MapReduce framework for analyzing next-generation DNA sequencing data. Genome Res 2010; 20: 1297-1303.

19 Murata Y, Yasumi T, Shirakawa R et al: Rapid diagnosis of FHL3 by flow cytometric detection of intraplatelet Munc13-4 protein. Blood 2011; 118: 1225-1230.

20 Schneider CA, Rasband WS, Eliceiri KWNIH Image to ImageJ: 25 years of image analysis Nat Meth 2012; 9: 671-675.

21 Frisan T, Levitsky V, Masucci M: Generation of lymphoblastoid cell lines (LCLs). Methods Mol Biol 2001; 174: 125-127.

22 Kunishima S, Hamaguchi M, Saito H: Differential expression of wild-type and mutant NMMHC-IIA polypeptides in blood cells suggests cell-specific regulation mechanisms in MYH9 disorders. Blood 2008; 111: 3015-3023.

23 Kunishima S, Kobayashi R, Itoh TJ, Hamaguchi M, Saito H: Mutation of the beta1tubulin gene associated with congenital macrothrombocytopenia affecting microtubule assembly. Blood 2009; 113: 458-461.

24 Travis MA, van der Flier A, Kammerer RA, Mould AP, Sonnenberg A, Humphries MJ: Interaction of filamin $A$ with the integrin beta 7 cytoplasmic domain: role of alternative splicing and phosphorylation. FEBS Lett 2004; 569: 185-190.

25 van der Flier A, Kuikman I, Kramer D et al: Different splice variants of filamin-B affect myogenesis, subcellular distribution, and determine binding to integrin [beta] subunits. J Cell Biol 2002; 156: 361-376.

26 Lad Y, Kiema T, Jiang P et al: Structure of three tandem filamin domains reveals autoinhibition of ligand binding. EMBO J 2007; 26: 3993-4004.

27 Pentikainen U, Jiang P, Takala H, Ruskamo S, Campbell ID, Ylanne J: Assembly of a filamin four-domain fragment and the influence of splicing variant-1 on the structure. J Biol Chem 2011; 286: 26921-26930.

28 Kiema T, Lad Y, Jiang P et al: The molecular basis of filamin binding to integrins and competition with talin. Mol Cell 2006; 21: 337-347.

29 Lad Y, Jiang P, Ruskamo S et al: Structural basis of the migfilin-filamin interaction and competition with integrin beta tails. J Biol Chem 2008; 283: 35154-35163.

30 Sheen VL: Filamin A mediated Big2 dependent endocytosis: from apical abscission to periventricular heterotopia. Tissue barriers 2014; 2: e29431.

31 Guerrini R, Dobyns WB: Malformations of cortical development: clinical features and genetic causes. Lancet Neurol 2014; 13: 710-726.

32 Antonucci A, Fronzoni L, Cogliandro L et al: Chronic intestinal pseudo-obstruction. World J Gastroenterol 2008; 14: 2953-2961.

Supplementary Information accompanies this paper on European Journal of Human Genetics website (http://www.nature.com/ejhg) 